\title{
DE LA GEOMETRÍA ALTERNATIVA A LA NUEVA GEOMETRÍA DE JOAQUÍN GONZÁLEZ, QUINO
}

\section{FROM ALTERNATIVE GEOMETRY TO NEW GEOMETRY OF JOAQUÍN GONZÁLEZ, QUINO}

\author{
Andrés LuQue Teruel \\ Universidad de Sevilla. España \\ luquete@us.es
}

\begin{abstract}
Joaquín González, Quino, es un pintor singular por su versatilidad y el extraordinario talento mostrado en amplias series, muy distintas en aspectos técnicos, desarrollos formales y estilos derivados de estos. Una de esas series, titulada Geometría Alternativa, lo llevó a finales de los años setenta y el primer lustro de los ochenta a la experimentación de la collografía, resuelta con planos que configuran espacios y volúmenes sintéticos, con los que determinó nuevas realidades artísticas y se quedó a un paso de la abstracción. Pasados algo más de veinte años, partió de esos principios para el desarrollo de una nueva serie, Nueva Geometría, resuelta con otro procedimiento, la pintura sobre lienzo, y en tamaños superiores. En esta nueva serie llegó a la completa abstracción, en dos variantes correlativas: en la primera, con planos y espacios resultantes resueltos con recursos táctiles; en la segunda, con planos con colores sin apenas o ninguna gradación cromática y texturas lisas.
\end{abstract}

Palabras claves: vanguardias; Quino; pintura abstracta; pintura geométrica; postmodernidad.

In the late Seventies and early Eigthies of the $20^{\text {th }}$ century, Joaquín González Quino produced a series of works -most of them technical collographs- in which he resolved urban or semi-rural perspectives through geometrically synthetic planes connected with the help of superpositions and juxtapositions. With them, he started an alternative plan to the painting of his day. The old textures of the collographs, authentic bas-reliefs with woods fitted together and organic materials that provide complex and dense surfaces, now correspond to such a delicate treatment of the planes. These simplified and minimal planes, both in the superficial demarcation and their physical nature, become known through the established relations as a whole. This is not only determining but also an indispensable ally to keep essential reference to visual reality. This New Geometry is the work of a mature and creative artist who initiated an own and innovative line in the art of his day, as it happens with the good painting, with the true art, capable of resisting the passing of decades and the mentalities that created it, and able to show itself impetuously on the intellective sphere of the current art. This, Quino, is an unquestionable reference, an artist with personality and own point of view, who is already imitated by young artists interested in soul rising through painting.

Keywords: avant-garde; Quino; abstract painting; geometric painting; postmodernity. 


\section{INTRODUCCIÓN}

Joaquín González, Quino, es un pintor de origen extremeño, formado y activo en Sevilla desde hace cuatro décadas. En ese tiempo ha luchado de modo incansable por darse a conocer, por abrirse un hueco en el contexto de la pintura sevillana ${ }^{1}$. Para él nada ha sido fácil en un mercado bajo mínimos y, a veces, simplemente, inexistente ${ }^{2}$. Su increíble imaginación nunca se doblegó a los límites materiales ni a ningún tipo de circunstancia adversa, todo lo contrario, a mayores dificultades, mayor aliento para un talento ilimitado, muchas veces desbordado por su propio ímpetu y, en las ocasiones, necesarias también, en las que la fatiga hacía ver el final de una etapa, capaz de reinventarse con nuevos argumentos e idéntica pasión ${ }^{3}$.

Cada vez que lo hizo fue con una serie completa, la mayoría de las veces, muy amplia, con tantas variantes formales que el concepto representativo y el propio tema quedaban agotados, al menos en lo que refiere a la identidad plástica de las relaciones internas que las fundamentan. Casi nunca tituló una obra concreta y cuando lo hizo fue por la circunstancia de una exposición individual o colectiva en la que la exhibió al margen de las demás de su misma serie, entre las que adquiere un sentido específico como parte de un proceso. Pocas veces también dedicó una sola obra a un tema, caso en el que, como ocurrió con Pobre del cantor, en 2004, lo asimiló a los recursos técnicos, formales y expresivos de una serie, por lo general aquella que lo ocupase en ese momento, o, si la había dado por concluida, por alguna anterior o la previa a ese colofón $n^{4}$. En este caso, el desarrollo formal, el interés por la materia y sus texturas, y el contenido simbólico del protagonista y los objetos, remiten a la serie El saber y el tiempo ${ }^{5}$, desarrollada entre 1988 y 1993.

${ }^{1}$ ANTOLÍN PAZ, Mario (ed.): "Joaquín González", en Diccionario de pintores y escultores españoles del siglo XX. Madrid, 1994, p. 1753; y APG: "Joaquín González”, $A B C$, Sevilla, 11 de enero de 1990, p. 41.

2 LORENTE, Manuel: "Mácula", $A B C$ de Sevilla, 5 de marzo de 1987, p. 74; HERRERA, Celia: "Joaquín González", Hoy de Extremadura, 1997, p. 68; MONTOYA, José Luis: "Joaquín González", Hoy de Extremadura, 1997, p. 68; PAVÓN, Víctor: "Joaquín González", Hoy de Extremadura, 1997, p. 68; HERNÁNDEZ, Mario: "Joaquín González”, La Capital, 1999, p. 68; VIGARIO, David: “Joaquín González”, La Capital, 1999, p. 50; PONCE DE LEÓN, R.: “Joaquín González”, El País, 1999, p. 70; y DE LA TORRE AMERIGHI, Iván de la: "Atávicas danzas", ABC Cultural, 29 de diciembre de 2001, p. 22.

${ }^{3}$ GÓMEZ PIÑOL, Emilio: Ruptura vanguardista, desintegración y nostalgia del Arte. Sevilla, 2005, pp. 64-ss.

${ }^{4}$ LUQUE TERUEL, Andrés: Joaquín González (Quino). Pobre del cantor. Sevilla, 2004, pp. 5-31.

5 LUQUE TERUEL, Andrés: “Joaquín González (Quino). Fundamentos teóricos de siete series inéditas", Laboratorio de Arte, 14, 2001, pp. 143-145. 
Esto no quiere decir que esas obras no tengan un argumento y un peso formal y técnico específico, por sí mismas, que lo tienen y permite apreciarlas como tales, sino que esa realidad interna, esto es, su ser obra de la obra ${ }^{6}$, tiene una lectura en sí misma y otra en el conjunto de la serie, como parte de la misma. De ese modo, a los contenidos formales, comunes en toda la serie, por lo tanto, genéricos en ese sentido, se les puede añadir una información específica, la que le corresponde a cada pintura en el momento determinado del desarrollo de la misma.

De esa manera, la imaginación de Quino no se vio coartada por los límites concretos de una sola obra, de las exigencias de un formato determinado. El punto de partida elegido le permitió presentar tantas variantes formales como su imaginación y las técnicas rápidas e intuitivas por las que siempre optó le permitían inventar sobre la marcha. Siempre fueron variantes plásticas sobre un mismo concepto de representación, que asoció al tema elegido de modo aleatorio, con una gran libertad y con el propósito de desarrollarlo desde sus argumentos técnicos y compositivos, propios y auténticos responsables del proceso creativo, nunca desde la dinámica que pudiese dictar cada tema en su narrativa habitual.

Puede decirse que, una vez elegido el planteamiento técnico y formal de la serie, este prevaleció sobre el tema, con independencia de que hubiera elegido antes a uno u otro. Para Quino, el tema fue casi siempre una excusa, un motivo para poder desarrollar un mundo figurativo fantástico, basado en las relaciones internas como argumento creativo prioritario y aun único, llegado el caso. Esas relaciones internas a las que Quino confió su creatividad estuvieron supeditadas a la aplicación de la materia, en el doble sentido de las texturas y los movimientos; los valores expresivos de los colores; y una particular incidencia de la luz.

Dilucidada la naturaleza de los argumentos creativos de Quino es necesario plantear otras cuestiones, relativas a las particularidades del proceso de ejecución. Francisco del Río dijo que Quino planificaba sus pinturas con valores muy próximos al diseño ${ }^{7}$. Ese es otro punto de partida que hay que tener en cuenta, sobre todo cuando se trata de construir o, más bien, de configurar, espacios, por arbitrarios e incluso imaginarios que pudiesen ser. En esos casos, la planificación es minuciosa, e inversamente proporcional a las libertades técnicas con las que suele interpretarlas y, de algún modo, simular ese punto de partida. La reflexión y el azar tienen en ello un protagonismo paralelo, un equilibrio sugerente, muy personal. En ese equilibrio se sustenta buena parte de su gran talento creativo.

La Geometría Alternativa fue una de esas series, una de las más personales y creativas y, seguro, la más intelectiva de toda su producción durante décadas. La desarrolló en el primer lustro de la década de los años ochenta del siglo XX en

${ }^{6}$ HEIDEGGER, Martin: "El origen de la obra de arte", en Caminos del bosque. Madrid, 1988 , p. 57.

7 DEL RÍO, Francisco: I Muestra de pintores contemporáneos en la Alameda. Sevilla, 1998, p. 19. 
una particular técnica mixta con doble lectura. La Nueva Geometría puede considerarse una continuidad en el tiempo de aquella, ahora resuelta con otras técnicas, óleo sobre lienzo, y un formato de mayor tamaño. El fundamento conceptual es análogo; los resultados formales de las dos variantes de la nueva serie son muy distintos de aquellos y entre sí.

\section{EL POSICIONAMIENTO ALTERNATIVO DE LOS PRIMEROS DIBUJOS, UN PUNTO DE PARTIDA RECONOCIBLE EN EL DISEÑO}

El posicionamiento geométrico de Quino, en el primer lustro de los años ochenta del siglo XX, no fue consecuencia de su afiliación a ningún movimiento internacional, fuese abstracto o figurativo, y tampoco a la relación directa con un movimiento o estilo artístico determinado. Puede asegurarse que llegó ahí de un modo personal y alternativo, por evolución natural en la planificación de los espacios y las figuras y, aún más, por las relaciones establecidas directamente entre estos, en las figuraciones con las que inició su producción a finales de la década de los sesenta y durante los años setenta de ese siglo.

Puede intuirse en Carabelas, dibujo con el que participó en la Exposición de Primavera convocada por la Real Academia de Bellas Artes de Santa Isabel de Hungría de Sevilla, en 1968. En este representó los elementos naturales con una simplicidad extrema, casi esquemática. La reducción de las formas a siluetas y su colocación como planos en niveles sucesivos en sintonía con una concepción del espacio no representado como tal, anticiparon los criterios que, convenientemente fomentados, fundamentaron las composiciones de la Geometría Alternativa en años sucesivos.

$\mathrm{Su}$ interés es claro en ese sentido, pese a diferencias aún importantes en la concepción de las composiciones. La mayoría de esos dibujos son paisajes en los que multiplicó las posibilidades de comunicación en un nivel esencial supeditado a la capacidad de percepción y la posible empatía del espectador. Quino no creó pensando en este, sino en él mismo, en una búsqueda metodológica que le permitiese expresarse con criterio en función de sus posibilidades. No fue nada complaciente. Necesitaba la colaboración activa del espectador como sujeto perceptivo activo y, sin embargo, se distanció de este con el efecto irreal de composiciones ficticias en las que priman la vaciedad del espacio y la ausencia de movimiento, conceptos de los que dedujo un extraño y personal efecto de insonoridad. Sus composiciones se presentan como partes silenciadas de una realidad ajena a los mecanismos de la actividad cotidiana. Puede proponerse como una peculiar interpretación de los elementos de la naturaleza y los objetos como volúmenes definidos según las reglas de la geometría y relacionados mediante ritmos concretos que resaltan la quietud y el orden. Estas serán claves fundamentales de la próxima Geometría Alternativa. 
Es curioso que el concepto de sonoridad, que no parece posible, que no lo es en la realidad material de una pintura, dibujo o grabado, se manifiesta como tal en estas obras de Quino gracias a la nueva ficción sujeta por decisión propia a las directrices del plano. Lo mismo sucede con la sensación de movimiento, también fingido y producido por estímulos ajenos al fenómeno en sí, que, asimilado, revierte y estimula de nuevo a los sentidos generando el efecto ficticio del sonido.

Son componentes de la realidad que necesitan apoyarse en formas concretas para expresarse, y el artista moderó, dosificó o acentuó, según su conveniencia. Por ese motivo, los objetos que forman parte de esos dibujos, presentan apariencias posibles en el ámbito de la realidad; condición que relegan y aun pierden con la acción simultánea de los tonos pasteles, con los que se alejó con decisión del natural y, al mismo tiempo, de las posibilidades coloristas que pudiese ofrecer el ejercicio pictórico.

\section{LA DEFINICIÓN DE LA GEOMETRÍA ALTERNATIVA EN LOS GRABADOS DEL MUSEO DE ARTE CONTEMPORÁNEO DE SEVILLA Y MÁCULA}

La Geometría Alternativa de Quino está completamente definida en Composición I y Composición II, dos collografías del Museo de Arte Contemporáneo de Sevilla. Son dos paisajes urbanos configurados mediante planos sintéticos contrastados por sus valores táctiles. En estos planteó la relación entre los planos geométricos, alineados o yuxtapuestos, y las formas que pudiesen originar o no, según la función de cada uno. Un plano sin función, equivale en estas obras a un espacio vacío, lo que lo llevó a ver el vacío como un problema de la forma y, en consecuencia, a tener en cuenta la nada como forma.

Aunque no lo parezca a primera vista, los dos presentan grandes diferencias, pues en Composición I mantuvo las relaciones visuales de la escala natural, superadas en Composición II. Lo hizo con la división de la segunda composición en dos sectores, el inferior reducido a solo plano, que se presenta como una abstracción geométrica con la que trascendió el posible ejercicio de estilo mediante la habilidad técnica en el uso de un solo color: el negro y sus gradaciones en función de las intensidades cromáticas y de las texturas y sus comportamientos ante la luz; el superior con el paisaje esquematizado y reducido a sus formas puras, esto es, geométricas, supeditadas a una sutil fuga a la que se aplican los planos.

En las dos composiciones, la superposición de los planos permite la diversificación de los objetos mediante los contrastes de las texturas y las gradaciones que determinan, incluidos aquellos sin correspondencia inmediata en la escala visual. En Composición II, la división en dos sectores bien diferenciados supera tal grado de definición y obliga a deducir un desarrollo plástico común a dos fines que se complementan y que pueden dar lugar a nuevos pronunciamientos teóricos. 
Estos, enunciados con anterioridad, son la Teoría de los objetos y la Teoría de los lugares. En el primer caso, la realidad física de los objetos, esto es, los volúmenes que les corresponden, definidos en tanto que tales, asumen el protagonismo de las configuraciones, determinando las relaciones que pudieran definir a anular, indistintamente, los espacios. En el segundo, el protagonismo recae en la definición de estos con cualidades plásticas que pueden reproducir o no lugares reconocibles. En los dos casos, cuando renunció a la similitud con la escala real, la nada, sea mediante un plano sin significado alguno, el espacio irreconocible o la supresión del mismo mediante la presencia del objeto como protagonista único, $\mathrm{y}$, con estas opciones, la quietud y el silencio, mantienen una relación armónica con la totalidad de los ambientes que evidencian la fenomenología a la que pertenecen el movimiento y el sonido. Todo esto le permitió proponer y admirarnos con su máximo logro en la Geometría Alternativa: una Teoría personal del equilibrio.

En cuanto concepto, ello denota cierto paralelismo con el espacio de la conciencia de Morandi, con el que, por otra parte, no guarda la mínima la relación estilística. Quino llevó su posicionamiento hasta el extremo de convertir los paisajes, los exteriores, en un reducto íntimo tal los espacios interiores indefinidos que acogen a las equilibradas composiciones de este. Morandi es el pintor del equilibrio por la perfecta composición de los objetos inertes agrupados con naturalidad, estableciendo relaciones volumétricas que significan desde un punto de vista determinado, lo que implica un claro reconocimiento del espacio como elemento fundamental. En Quino el equilibrio radica en la armonía entre la forma y la vida y su compromiso con los posibles desarrollos formales. Así que la nada de un plano abstracto basado en sus posibilidades táctiles se integra en la totalidad de las formas con las que compone y en el paisaje del que forma parte o al que sirve como estímulo sensorial.

Las partes y los elementos de las composiciones de Quino adquieren una relación de equivalencia en la medida que cada textura tiene una función concreta y ello hace que visualmente los planos indeterminados en la escala humana signifiquen tal los objetos. Digámoslo de otra manera, el espacio vacío, el plano que no representa nada, responde a una condición táctil determinada, significativa en sí, en su realidad material, y esta, con independencia de la forma, sí es significativa en el sistema de relaciones plásticas que determina la configuración. De ese modo, la aparente nada de los pequeños planos asociados de Composición I y del espacio geométrico de Composición II, son elementos de los respectivos paisajes, pues cada textura, que en principio parecería ser la piel o el relleno de un simple tema o espacio, se convierte en protagonista y ello transforma la nada en objeto de naturaleza táctil y portador de una información plástica que radica en sus posibilidades como estímulo sensible.

La nada de esos planos que configuran Composición I y Composición II es, pues, un objeto plástico cuya forma manipuló el artista a su conveniencia y tal le 
proporcionó la riqueza de lo posible en su posibilidad ${ }^{8}$. En esto concuerda con la definición de la teoría del Taos de Chantal Maillard, relativa a un azar ontológico como actividad metafísica en la que se dan dos condiciones: el azar como Ley de la posibilidad y el Arte como conocimiento en su posibilidad. Esa equivalencia de Quino propició el singular conocimiento de lo no visible de esos paisajes mediante lo posible que insinúa el Arte, definido en consonancia con la actividad y las posibilidades perceptivas del sujeto cognoscente.

En esto debemos reconocer una riqueza que atañe tanto al artista como creador de formas en el infinito desarrollo de cada idea formal, como a las posibilidades de comunicación que conlleva cada una. Entendido así, la obra funciona en dos órbitas, una plástica que implica a lo visible y a lo táctil; y otra intelectual como metalenguaje que comunica mediante las sensaciones que producen tales estímulos, con las que se confirma el sentido opuesto al plano simple derivado de Mondrian. También debe distinguirse de los planos en apariencia neutros de Carlos Montaño, identificados por Francisco del Río como no lugar, pese al desarrollo pictórico equivalente y derivado del informalismo que le otorga identidad plástica ${ }^{9}$. Este planteó la forma de los no lugares como la parte de la estructura fragmentada con la que Carlos Montaño revisó la abstracción desde una perspectiva que aúna el legado pop y la nueva figuración, esto con una intención postmoderna con la que cuestionó la manifestación de la técnica y el estilo como expresión de una profunda subjetividad.

Como ha quedado expuesto, la intervención de los planos en las configuraciones de Quino tiene otro sentido. Su Teoría del equilibrio determinó otros principios figurativos asociados a la collografía técnica; sin embargo, es una teoría implícita en el desarrollo formal que corresponde reformular, pues la figura absorbe los mismos principios que aplica a las generalidades y a los planos que superan a la nada con su compromiso visual en la composición.

\section{EL RECONOCIMIENTO DE LA GEOMETRÍA ALTERNATIVA EN LOS PREMIOS INTERNACIONALES}

Quino participó en las exposiciones internacionales de la Academia Europea de las Artes, en las que obtuvo importantes galardones en 1985, con una serie de obras en técnicas mixtas, en las que hizo uso de los conceptos geométricos ensayados hasta ese momento, adaptándolos a distintos temas y espacios de representación más o menos reconocibles.

En esas obras, los planos son tales y también siluetas o hueco siluetas de figuras y manchas en blanco de las dos posibilidades. Hay que distinguirlo, pues

\footnotetext{
${ }^{8}$ MAILLARD, Chantal: La razón estética. Barcelona, 1998, pp. 65-80.

9 DEL RÍO, Francisco: Montaño. Sevilla, 1993, pp. 36-37.
} 
Quino obtuvo un gran rendimiento de esas asociaciones. El hueco silueta es un plano definido cuya plantilla no tiene equivalencia material, mas la determina sobre el fondo al que se aplica. En esa relación, las posibilidades fluctúan desde el desarrollo material o táctil del objeto o la figura que delimita en negativo y que, en ese caso, contrasta con la lisura o la mancha en blanco del plano o hueco silueta que la constituye, hasta el efecto contrario de un hueco silueta desarrollado en sus posibilidades táctiles sobre el fondo en blanco o de color liso sobre el que se crea el efecto de la silueta. En los dos casos, la silueta del objeto no existe, mas se ve y la valoración del negativo permite el ejercicio específico pictórico en el que se fundamenta el desarrollo del tema.

Las collografías de Quino son un claro ejemplo de la voluntad postmoderna, definida por la propia naturaleza de la técnica, derivada del collage y asumida como superación del mismo en función de nuevos fines, mas no como la aniquilación del procedimiento que concordaría con la voluntad de la modernidad. No es solo una cuestión técnica, también de forma, pues, al mismo tiempo, Quino asumió las fuentes en la definición del tema y en las variaciones.

Puede verse muy bien en los tres grabados premiados con la Medalla de Bronce en la Exposición Internacional de la Academia Europea de las Artes, celebrada en Bruselas, en 1985, titulados Amanecer, Mujer con sombrero y Desnudo. En el primero desarrolló la Teoría del equilibrio antes enunciada, en el segundo fue capaz de mantener las referencias del natural, y en el tercero de llegar a la esquematización más radical. La simplicidad de este es máxima, tanto en el desarrollo técnico como en el planteamiento formal.

Centrémonos en ese Desnudo, un solo plano sobre fondo somero sinuoso y desdoblado cuatro veces genera la silueta femenina, que presenta en un amplio fragmento del torso recostado y sin las extremidades ni la cabeza, con el doble foco visual de los senos y el pubis, cuyos volúmenes determinó con un rayado esencial, muy cuidado en el acabado de los perfiles, con el que no perdió las referencias volumétricas. La fragmentación del cuerpo femenino y su monumental simplificación mediante la fusión de los volúmenes, lo acercaron a la abstracción orgánica, de la que difiere al no asumir su valoración de la materia y sí la del natural. Ello lo llevó a una figuración esencial, dotada con un naturalismo extremo, que, en alarde de postmodernidad y de modo paradójico, acentuó con el recurso vanguardista de la multiplicación de los puntos visuales al enfocar uno de los senos desde abajo y de perfil y el otro desde arriba para indicar su caída. Ese recurso o licencia artística fue fundamental. Las gradaciones cromáticas y las texturas crean los efectos anatómicos complementarios y la tintada monocroma tiene matices simbólicos, ya que el fuerte claroscuro de la versión original en blanco y negro alude a las posibilidades sexuales de la mujer, en consonancia con la tradición de la Venus clásica, y la versión en verde a la esperanza puesta en ella como madre, según la Madre Tierra de raíces prehistóricas. La simplicidad equivale a las atractivas apariciones de Félix de Cárdenas, dotadas también de un contenido 
simbólico cuyo valor intelectivo supera a la aparente ingenuidad, mas difiere de este en la valoración de lo específico pictórico y en los recursos con los que opera.

Una collografía muy representativa de la Geometría Alternativa de Quino es la titulada El contrabajo, galardonada con una Mención de Honor en la Exposición de la Academia Europea de las Artes, celebrada en París, en 1985. La Teoría del equilibrio adquirió aquí un doble sentido. El primero de ellos refiere a los planos y a los objetos mediante la nada que supone la mancha en blanco del plano que alude al músico y alberga a la base del instrumento que toca, que, por el corte cóncavo de ella y el hueco silueta que forma y acoge al espacio, sale del interior y supera a esa nueva forma semioval y transida por el aire y, pasando tras el arco, a la segunda fuga lineal y aérea del ventanal del fondo, para superponer el mástil a la mancha en blanco y ocupar su cabeza. Ese desarrollo formal tiene un claro valor simbólico: la música procede del hombre y no del instrumento. La ambivalente mancha en blanco a modo de silueta y de hueco silueta significa como estímulo sensible y opera sobre la silueta a la que alberga y complementa. Tales relaciones entre las siluetas y ellas en el negativo del hueco crean unos efectos espaciales valorables en sí y respecto del espacio total que los acoge, en el que rige una doble fuga lineal: la de la estancia guiada por el pavimento y la del ventanal de fondo que abre una amplia perspectiva. Ahí entra en juego el segundo sentido propuesto, pues el instrumento musical ejerce de eje visual que simboliza la responsabilidad del músico en relación con la música que genera, y, en consonancia, a las acciones y las intervenciones del hombre y su responsabilidad en la vida social. Este potencia y refuerza al primero, al que no sucede sino con el que actúa de modo simultáneo. Las equivalencias, mutuas, avalan la Teoría del equilibrio que rige su creatividad. El músico y el contrabajo son el nexo de los dos sentidos, pues ahora el equilibrio se establece mediante las valoraciones táctiles y lumínicas que proporciona la collografía y matiza la compensación de los planos, los espacios y los objetos y sus cualidades físicas relativas al movimiento, la sonoridad y la luminosidad.

Ese doble equilibrio magnifica la teoría con su magistral unicidad, capaz de manifestarse con la misma intensidad en la dimensión estética, de índole plástica y contemplativa, y en la dimensión simbólica, con su alcance intelectivo. Es un ejemplo perfecto de postmodernidad, debido a la aplicación de la técnica vanguardista con un sentido sintético, pleno de creatividad, con el que planteó un símbolo actual, que remite y recrea la vieja idea barroca del buen gobierno admitido por todos, ahora como gobierno de las intenciones propias de cada espectador en relación con el compromiso adquirido en la vida en comunidad. El contrabajo es una declaración de las intenciones de la libertad atenta a las obligaciones sociales, que representan la nobleza del músico y el instrumento y su grandeza y su soledad en el eje que constituyen como primer plano y núcleo de la composición. 
En la configuración de esa obra, las posibilidades plásticas son determinantes para el hecho contemplativo o estético y para el mensaje simbólico. La versión original en blanco y negro otorga un valor preeminente a la mancha en blanco del plano que configura al contrabajista y el hueco silueta que, de ese modo, interviene en la figuración y, a la vez, parece horadar el plano con un sentido etéreo análogo al de los grupos escultóricos de Enrique Ramos Guerra, en los que, según Juan Ramón Barbancho, mostró una clara obsesión por captar la esencia del hombre, que lo llevó a las figuras vacías con las que buscó la libertad y reflejó la angustia ante lo desconocido ${ }^{10}$. En tiradas sucesivas y en color, la última en 1998, las sutiles y óptimas y brillantes gradaciones cromáticas generan nuevos estímulos y ellos otras posibilidades de percepción, pues modifican las valoraciones plásticas y también los estados de ánimo y los sentimientos que nos implican en el símbolo o determinan nuestra relación o grado de aproximación a este.

Otro nivel de reconocimiento le llegó con la celebración de Mácula, organizada por la Sección de Arte del Aula de Cultura de la Facultad de Geografía e Historia de Sevilla ${ }^{11}$, en la que también participó el pintor de Granada Juan Carlos Ramos, en 1987. Las obras que expuso aquí son muy representativas de la Geometría Alternativa, mayoritariamente resuelta mediante la collografía técnica ${ }^{12}$, las más importantes El contrabajo, El campesino, Venus y El pueblo. En El campesino, además de la organización somera del espacio y el valor geométrico de los planos, destaca una abstracción lineal como parte superior de la silueta figurativa. En Venus, el hueco silueta produce un bello desnudo mediante la mancha en blanco que radicaliza la simplificación anatómica. Más compleja es la concepción de El pueblo, un paisaje en el que superpuso dos grandes planos, el de los contornos rurales y el del núcleo urbano reducido y simplificado, que se relaciona con los anteriores por un lirismo matizado por el color, sea en blanco y negro o en tonos pasteles de colores complementarios tales rojos y verdes o acentuados por la melancolía de los ocres combinados. En definitiva, el desenfado de la integración del elemento abstracto en la configuración de El campesino; Venus, con su hueco silueta único y con su comunicación directa; y El pueblo, con su lírica superposición de planos, son, junto a El contrabajo, ejemplos representativos de la Teoría del equilibrio de Quino. Como él mismo reconoció, pensó imprescindible compensar el volumen y el espacio, la composición y el objeto, el dibujo y el color, la forma y la expresión ${ }^{13}$.

${ }^{10}$ BARBANCHO, Juan Ramón: Enrique Ramos Guerra. Sevilla, 1997, p. 9.

${ }^{11}$ La exposición se celebró en el Pabellón de Uruguay entre el 23 de febrero y el 7 de mayo de 1987.

12 ANTOLÍN PAZ, Mario Antonio: “Joaquín González”, op. cit., p. 1753.

${ }^{13}$ Correspondencia del artista. 


\section{UN NUEVO GIRO HACIA UN EXTREMO OPUESTO: LA CULMINACIÓN DE UN CAMINO PROPIO CON LA SERIE NUEVA GEOMETRÍA}

Pasados veinte años de aquellos planteamientos geométricos tan personales, hacia 2005, Quino reparó en ellos como punto de partida para el desarrollo de una nueva serie, titulada Nueva Geometría, en la que potenció los argumentos iniciales, prescindiendo por completo de cualquier elemento que pudiese distraerlo de ello ${ }^{14}$. Esa fue una diferencia fundamental, pues en estas nuevas obras Quino se dedicó en exclusiva a la configuración de espacios, virtuales e intelectivos, en los que no tuvo cabida ninguna actividad humana ni más valores simbólicos que los relativos a la representación del espacio; otra, no menos significativa, el cambio de soporte y técnicas de ejecución, pues pasó de las técnicas mixtas propias de las collografías a la pintura con óleos sobre lienzo; y también de los formatos reducidos o de dimensiones medianas a los de gran tamaño. Esto coincidió con su intervención como comisario de la exposición Vigencia de las Vanguardias en la pintura sevillana, celebrada en la Galería Concha Pedrosa de Sevilla, en 2007, en la que participaron algunos de los más destacados protagonistas del arte contemporáneo en la ciudad ${ }^{15}$.

A diferencia de aquellas obras con las que obtuvo reconocimiento internacional en la década de los ochenta, en estas pinturas de la Nueva Geometría la frontera entre la figuración simplificada al máximo y la abstracción es mínima, sutil, casi imperceptible, sobre todo en aquellos casos en los que la estructura se fundamenta exclusivamente en el valor constructivo de los planos, siempre racional y, por lo tanto, tendente a la desnudez de las figuras geométricas u otras asimétricas complementarias entre sí y dispuestas en régimen de equivalencia. Incluso puede decirse que, minimizada o eliminada la referencia natural común, esas obras que traspasan los límites se presentan como pinturas abstractas en el amplio sentido del término. Es otra gran diferencia con la Geometría Alternativa, en la que realidad se mantuvo siempre presente y bien visible, pese a las simplificaciones más extremas.

La Nueva Geometría es, además, una serie mucho más amplia que la anterior. Las pinturas abstractas en formato grande y mediano, remiten a los planteamientos conceptuales y racionales de las collografías de varias décadas antes, llevados

${ }^{14}$ LUQUE TERUEL, Andrés: Vigencias de las vanguardias en la pintura sevillana. Sevilla, 2007, pp. 110-112.

${ }_{15}$ Miguel Pérez Aguilera, José Luis Mauri, Luis Gordillo, Paco Cuadrado, Francisco Cortijo, Gerardo Delgado, Manuel Salinas, Marina Díaz Velázquez, Juan Fernández Lacomba, Rolando Campos, Guillermo Pérez Villalta, Pedro Simón, Carlos Montaño, Horacio Hermoso, Carmen Rufo, Juan José Fuentes, Rafael Zapatero, Manolo Cuervo, Paco Broca, José Manuel Pérez Tapia, Álvarez Fijo, Alberto Donaire, Ricardo Castillo y Rorro Berjano. 
ahora a una situación extrema, a un punto de simplificación radical que las pone al límite y aun determina la superación de la figuración en beneficio de la configuración abstracta derivada. La creatividad y la originalidad de estas obras lo sitúan en un nivel muy personal dentro de la abstracción, en la que destaca por la economía de medios y del lenguaje formal y la asunción de inquietudes informalistas en tal proceso, en principio antagónico. Las viejas texturas de las collografías, auténticos relieves con maderas ensambladas y materias orgánicas que proporcionan superficies complejas y densas, tienen ahora correspondencia en sutiles tratamientos de los planos que, simplificados, mínimos, tanto en la acotación superficial como en su naturaleza física, significan mediante las relaciones de conjunto establecidas ${ }^{16}$. La reducción del color es pareja y la intensidad de la luz, determinante, una aliada imprescindible para mantener la referencia mínima necesaria con la realidad visual ${ }^{17}$.

Los planos geométricos, reducidos a la mínima expresión, están resueltos con óleos y sutiles empastes y veladuras en las dos variantes que presenta esta primera etapa o fase de la serie, una con gamas ocres y reflejos de óxidos; la otra, en blanco y negro con la mediación de grises y, en algunos casos, con algún leve toque lineal de color.

Rafael Cerdá, detectó en estas pinturas de Quino una clara evolución desde las obras fundamentadas en los valores táctiles y con colores sombríos, consecuencia estos de épocas y series anteriores, hasta las pinturas en las que optó por estructuras geométricas severas, en las que los planos prescinden de los recursos expresivos de las texturas, sustituidas por colores contrastados, con los que consigue el equilibrio y la armonía necesaria para inducirnos a composiciones sugerentes y muy serenas a la vez ${ }^{18}$.

En definitiva, la Nueva Geometría de Quino es la obra de un artista maduro y creativo, que inició una línea propia aunando con criterio tendencias significativas de su tiempo, como sucede con toda la buena pintura, con el arte verdadero, capaz de resistir el paso de las décadas y las mentalidades que lo generaron y de proyectarse de nuevo, con fuerte ímpetu, en la esfera intelectiva del arte actual. La vigencia de los planteamientos y la actualización del lenguaje expresivo avalan la serie como una de las aportaciones más personales e interesantes de la pintura española actual.

Con esta propuesta, Quino se presentó como una referencia indiscutible, como un artista con personalidad y criterios propios, imitados ya por jóvenes artistas interesados en la elevación intelectiva mediante la pintura. En un momento en el que la

${ }^{16}$ LUQUE TERUEL, Andrés: Grupo Pegamento. Punto de encuentro. Sevilla, 2013, pp. 69-72.

${ }^{17}$ LUQUE TERUEL, Andrés: "Geometría Alternativa", en Galería Cristóbal Bejarano de Linares y Arte Informado. Linares, 2011, s. p. Vid. http://www.arteinformado.com/ agenda/f/geometria-alternativa-47807 (Consultado el 15-10-2017).

18 CERDÁ, Rafael: "Geometría Alternativa”, en Galería Cristóbal Bejarano de Linares y Arte Informado. Linares, 2011, s. p. Vid. http://www.arteinformado.com/agenda/f/ geometria-alternativa-47807 (Consultado el 15-10-2017). 
mayoría de los jóvenes pintores deambulan sin criterios definidos o se aferran a las variantes estilísticas de caminos preestablecidos por otros, o, lo que es más frecuente, a la indefinición de la tendencia denominada mala pintura, sin parámetros personales que la fundamenten, Quino tuvo el valor de volver sobre la abstracción geométrica que había practicado en su juventud con la seguridad de sacarle mayor provecho con otros procedimientos y a mayor escala, aumentado el grado de dificultad.

En algunas pinturas intensificó la abstracción y redujo la incidencia de los colores sobre los planos básicos; y en otras introdujo claves simbólicas, como en $\mathrm{La}$ casa de Ali, en 2015, en la que la interacción de los planos y las letras ajustadas a estos presentan una doble lectura, formal y fonética. La síntesis de recursos de esta obra, con una hábil combinación de superficies lisas y sintéticas y otras táctiles y veladas; así como de colores cálidos, entre las primeras, y otros fríos, indica el sentido de un nuevo camino.

\section{LA OPCIÓN SINTÉTICA DE LA NUEVA GEOMETRÍA}

La definición y la evolución natural de la Nueva Geometría llevaron a Quino a una variante significativa de la serie desde un punto de vista plástico. Esta quedó expuesta de modo claro con la pintura antes citada, una de las pocas con un título específico, debido a un encargo concreto.

Consistió en la configuración de espacios ficticios mediante la intensificación de la abstracción y la reducción radical de la incidencia de las texturas sobre los planos básicos, lo que, por otra parte, potenció un nuevo tratamiento del color, en la mayor parte de las superficies plano, sin gradaciones cromáticas o efectos mínimos en ese sentido ${ }^{19}$. La incidencia del color pasó a tener un nuevo protagonismo y la relación de los planos se estableció en un nivel de relaciones internas en las que la realidad visual quedaba definitivamente reducida al nivel de un recuerdo lejano, de una semejanza vaga, de una asociación por empatía. Por ese motivo, prescindió de la figura humana e incluso de la definición de los volúmenes como objetos reconocibles, pues, en ese caso, la simplificación procedería sobre principios orgánicos, y el paso hacia la abstracción encontraría un obstáculo en la empatía del espectador que establece relaciones de modo espontáneo.

Eso sí, la simplificación de la Nueva Geometría llevó a Quino a una posición afín en una serie figurativa, Anatomía básica, firmada en 2011, aunque esta responde a otros principios, basados en la conciencia de la anatomía. Lo que hizo fue simplificar el cuerpo femenino al máximo, despojándolo de cualquier accesorio, fuese del propio cuerpo o añadido. Cualquiera sabe que ningún cuerpo tiene

${ }^{19}$ LUQUE TERUEL, Andrés: Grupo Pegamento. Convergencias y divergencias. Sevilla, 2014, pp. 22-25; y LUQUE TERUEL, Andrés e IGLESIAS CUMPLIDO, Alicia: Grupo Pegamento. Luz, materia y forma. Figueira da Foz, 2016, pp. 26-35. 
nada accesorio, que todo es parte del mismo y tiene alguna función, por complementaria que sea. ¿Qué es, entonces, accesorio para él en esta serie en el cuerpo de una mujer? La respuesta es sencilla, cualquier rasgo que la personalice, que permita identificar o tan siquiera imaginar una entidad. Su propuesta es la de un cuerpo femenino genérico, en su plenitud carnal, recio, voluptuoso, sensual, erótico. Para representarlo tuvo bastante en todos los casos con una parte del mismo, casi siempre comprendida entre las rodillas y la base del cuello. En el procedimiento hay una clara analogía con la alusión a espacios compartimentados, a planos combinados en una serie de combinaciones basadas en la probabilidad.

El trabajo inicial con plantillas de cartulina recortadas sobre un fondo de otro color, procedimiento elevado a la categoría artística por Marcel Duchamp en el primer cuarto del siglo XX, le permitió resolver las figuraciones con siluetas planas, en las que las líneas de contorno expresan la realidad física de los cuerpos desde una perspectiva general y genérica. Cada una de esas plantillas es análoga a los planos de los paisajes sintéticos, irreales e imaginarios, de la Nueva Geometría. A diferencia de aquel, una vez fijado tanto en estos como en las figuraciones su propósito y el sentido de la simplificación esencialista de los espacios inventados o sugeridos y del cuerpo femenino, con el consecuente énfasis de los rasgos de estos, Quino los utilizó como referencias para pintar lienzos en los que reprodujo tales condiciones.

En esos lienzos utilizó una combinatoria de planos presentados con un sentido del orden derivado del diseño, y con la capacidad suficiente para determinar espacios; procedimiento equiparable a las siluetas resueltas con tintas planas y las siluetas previas de papel recortado sobre idéntico soporte, análogas, que muestran cuerpos femeninos que oscilan entre la abstracción y la sensualidad de las formas, en los que dejó claros sus gustos personales, como artista. Es, por lo tanto, antes que nada una cuestión de procedimiento, que, después y en segunda instancia, tiene un vínculo formal preciso en el desarrollo de dos series distintas por su naturaleza en función del objeto de representación, el espacio, tratado mediante planos; y el volumen, reducido a la condición plana de la silueta.

La simplicidad de los procedimientos y de las formas puede aparentar un interés menor por ambos, sensación errónea, pues la pureza de los planteamientos esencialistas entraña siempre una enorme dificultad. No es fácil convertir un espacio o una forma reducida a su mínima condición formal en una configuración dotada con expresión artística, y Quino lo consiguió, en buena medida por su magisterio compositivo y en parte también porque la fijación de un espacio o la definición de un cuerpo y, por extensión, las siluetas que los determinan, nunca son sencillas y siempre contienen una información visual con atractivos suficientes para la percepción de cualquier persona y, por supuesto, para la interpretación y las sucesivas reinterpretaciones del artista.

Fecha de recepción: 30 de octubre de 2017

Fecha de aceptación: 12 de abril de 2018 


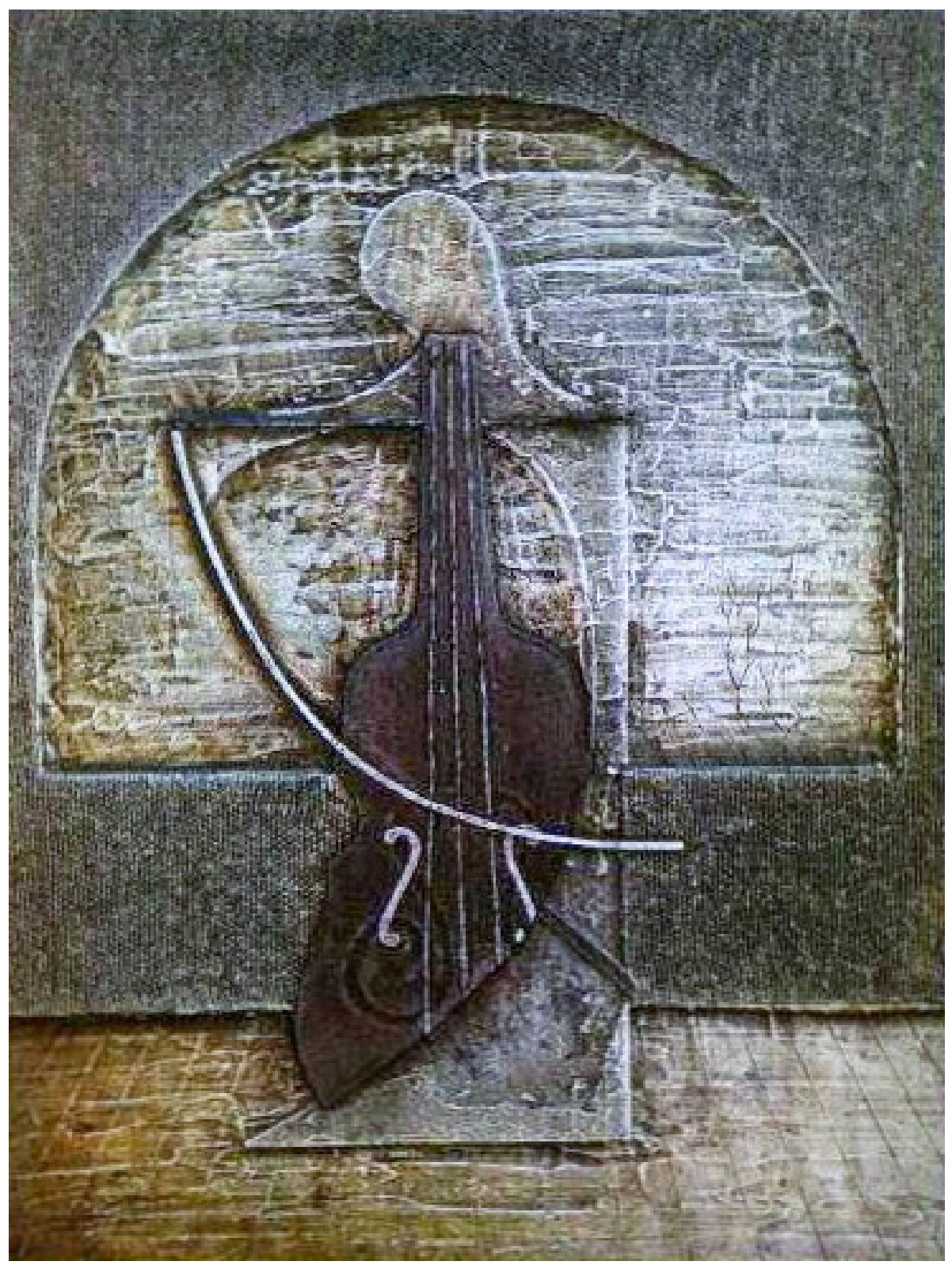

Figura 1. Quino, El violoncello, 1985.

LABORATORIO DE ARTE 30 (2018), pp. 475-496, ISSN 1130-5762 e-ISSN 2253-8305 - DOI http://dx.doi.org/10.12795/LA.2018.i30.26 


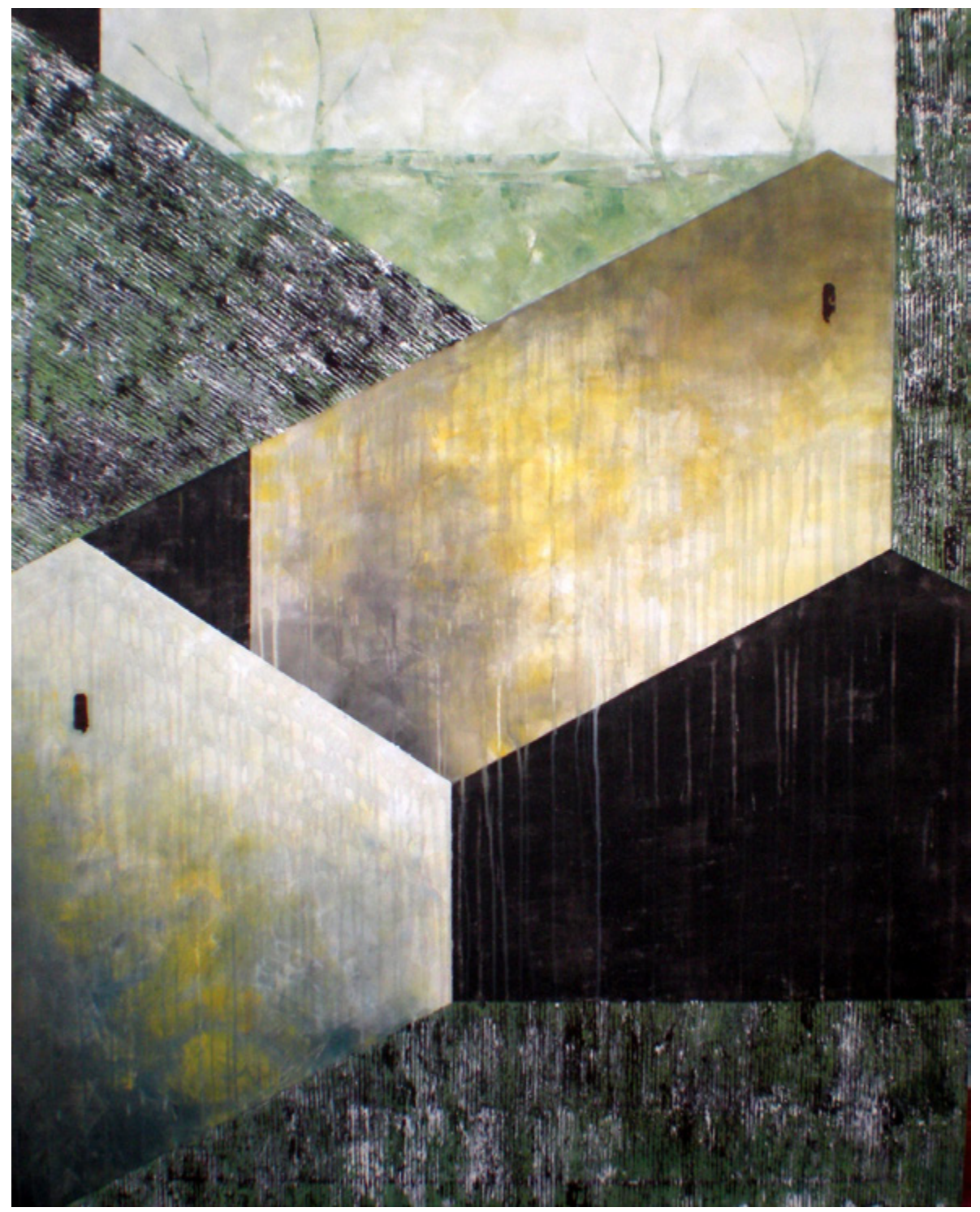

Figura 2. Quino, Nueva Geometría, 2010. 


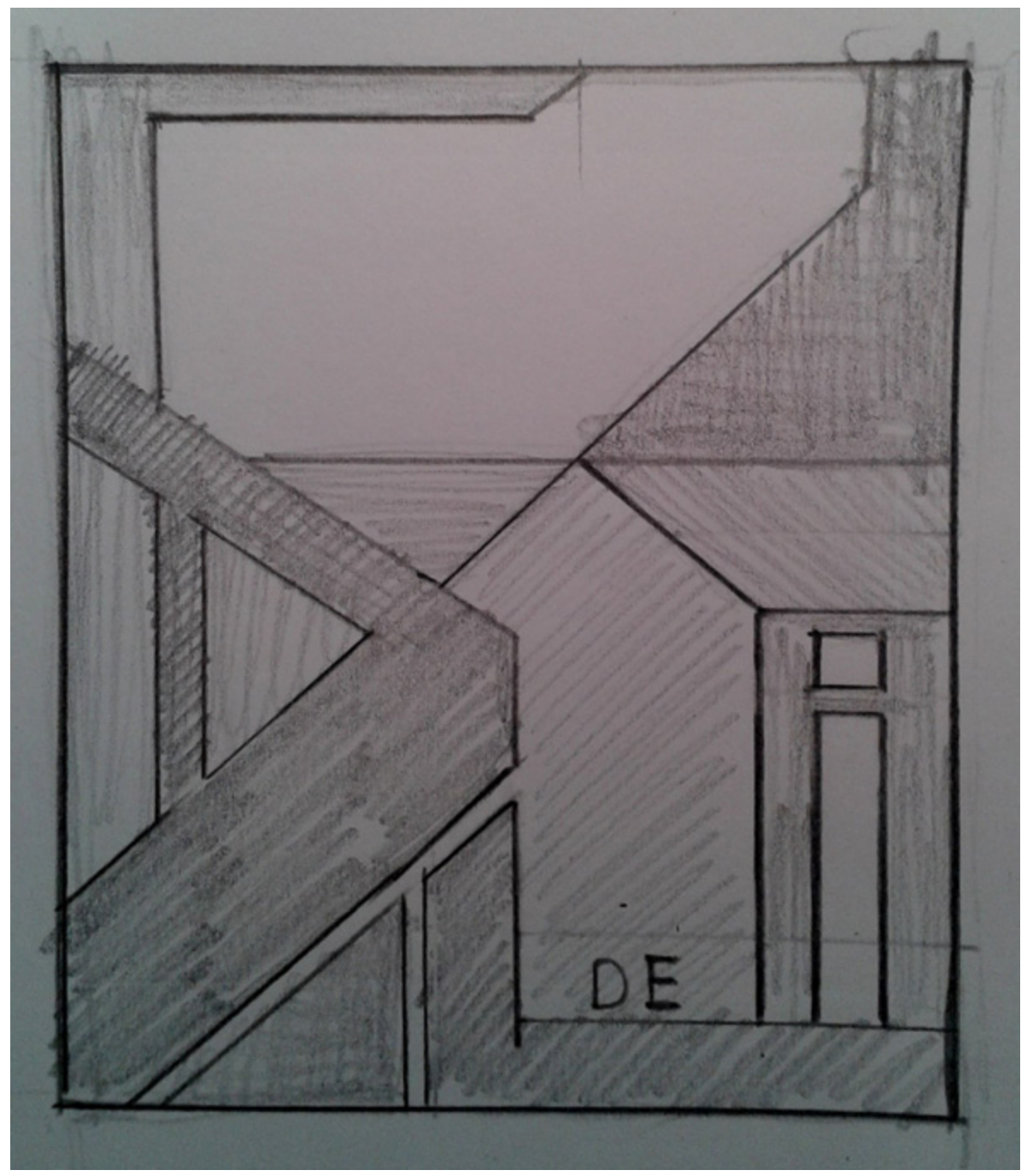

Figura 3. Quino, La casa de Ali, 2015. 


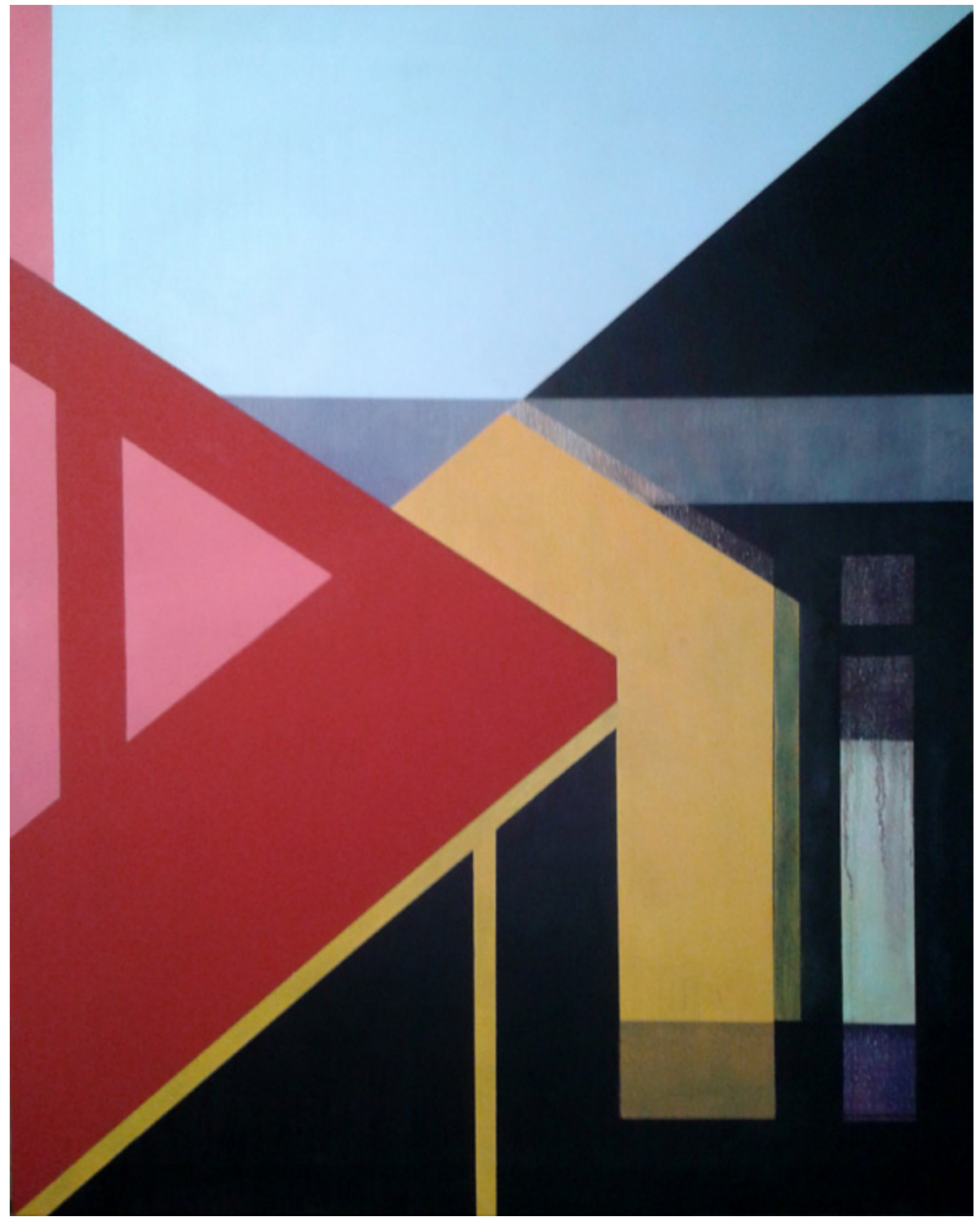

Figura 4. Quino, La casa de Ali, 2015. 


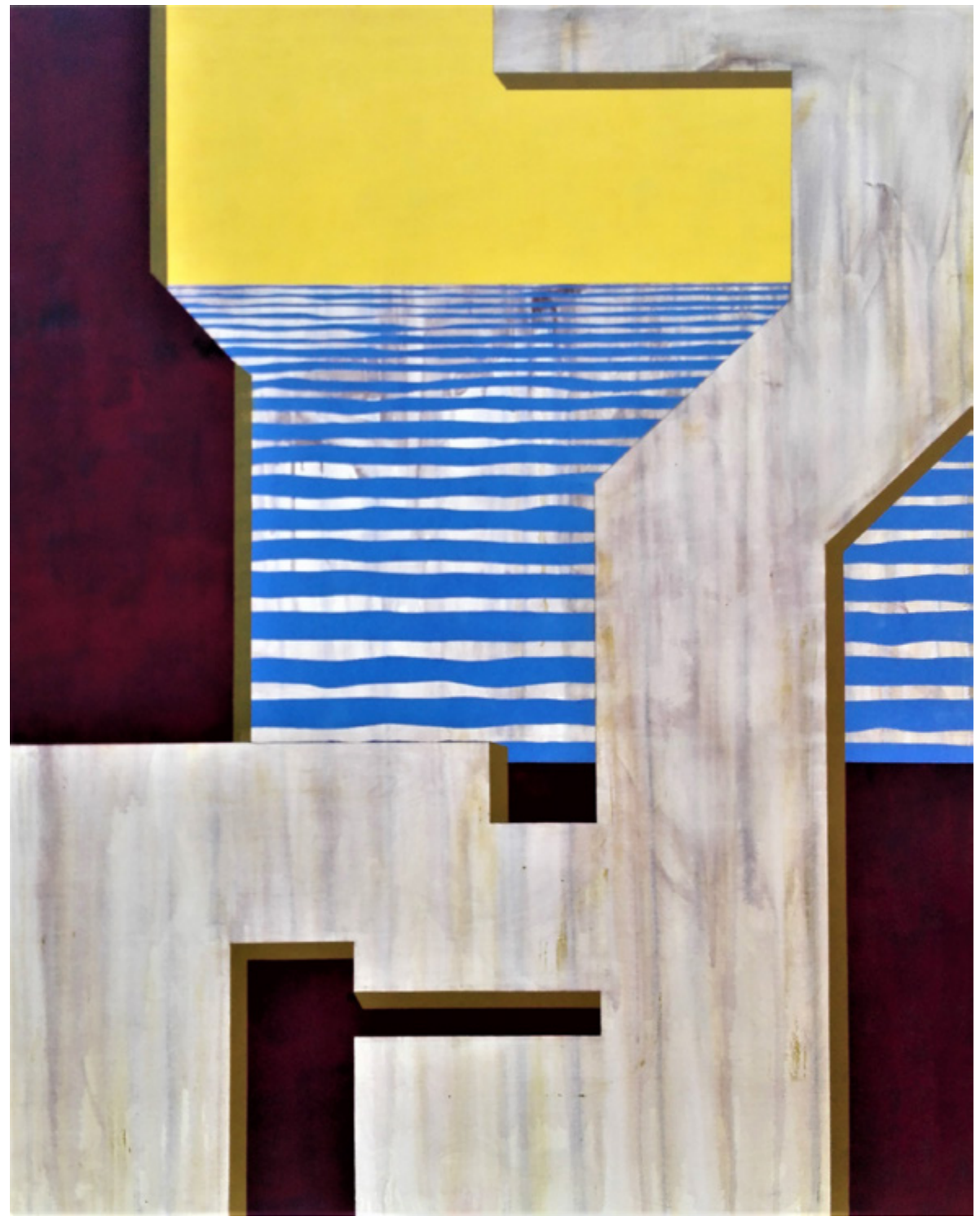

Figura 5. Quino, Nueva Geometría, 2016. 


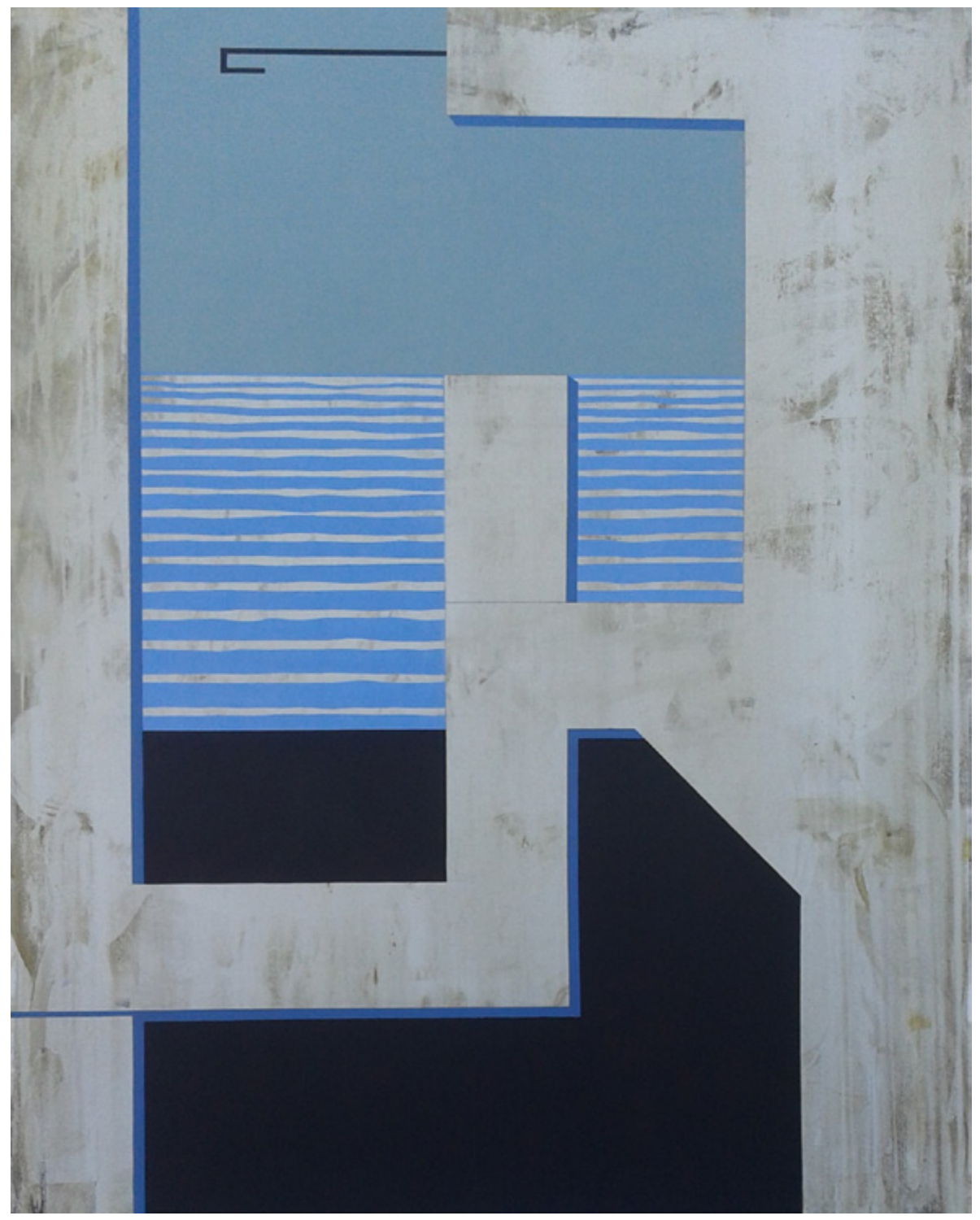

Figura 6. Quino, Nueva Geometría, 2016. 


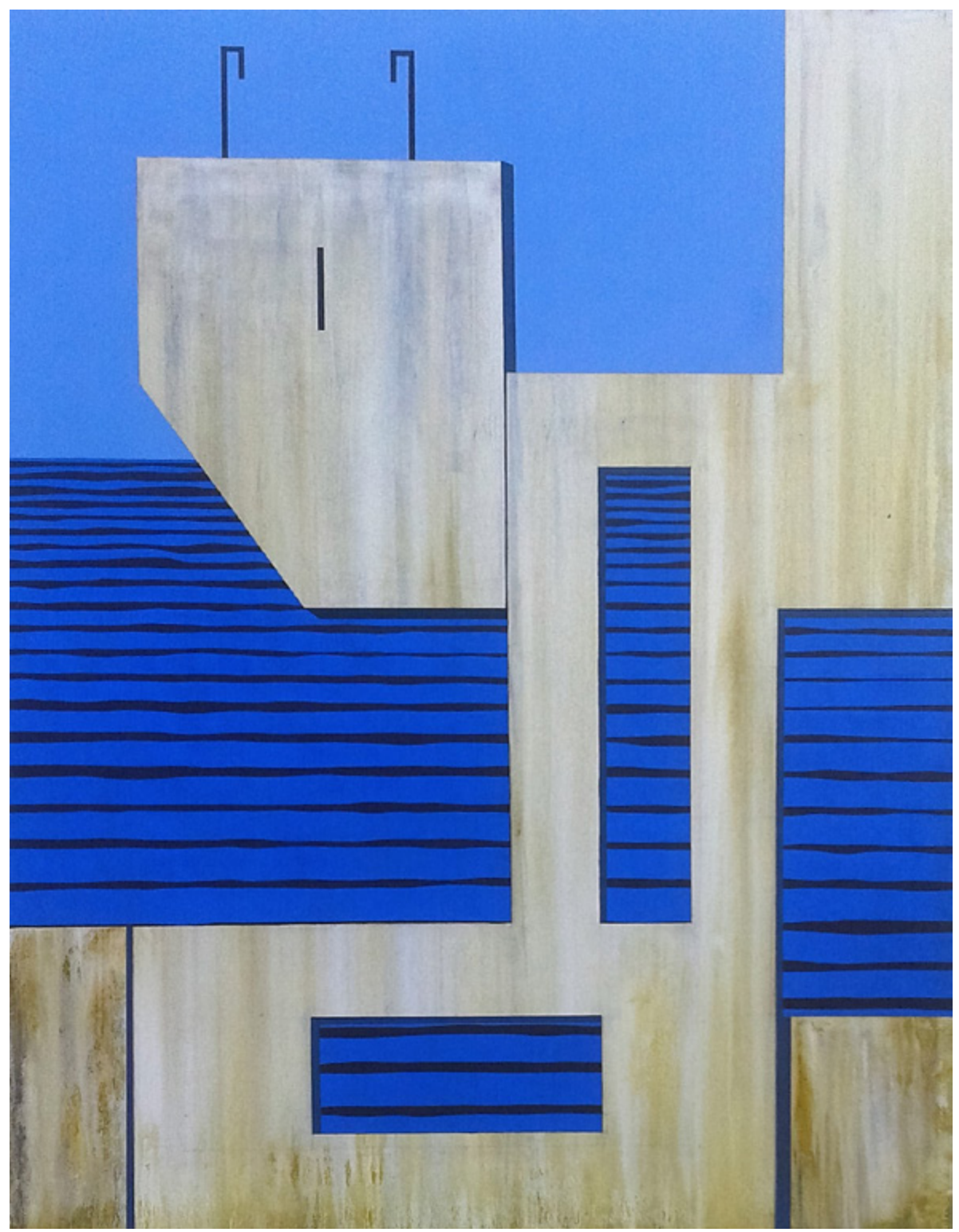

Figura 7. Quino, Nueva Geometría, 2016. 


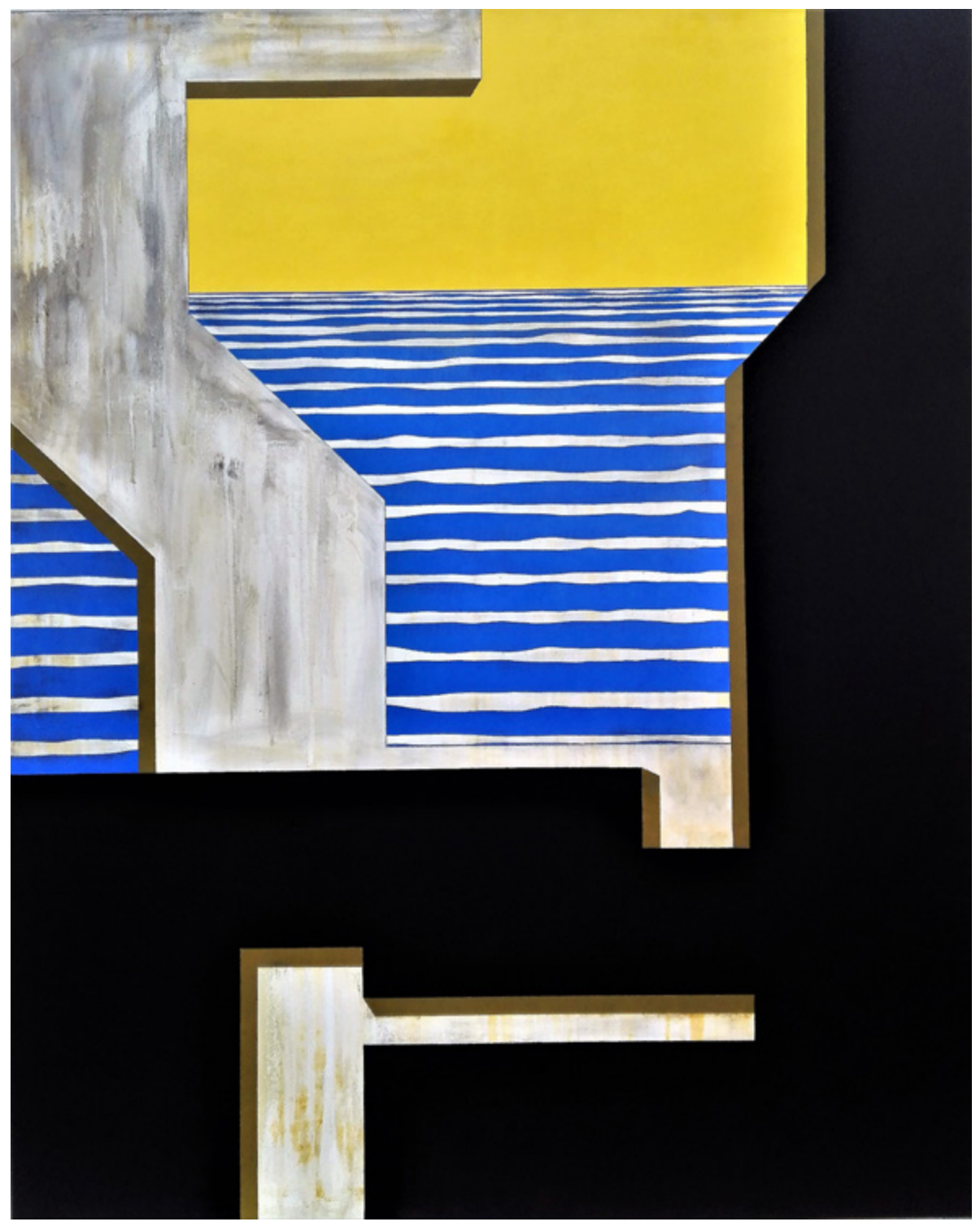

Figura 8. Quino, Nueva Geometría, 2017. 